\title{
Response properties of nucleus reticularis lateralis neurons after electroacupuncture stimulation in rats.
}

Kentaro Moritaka $^{1}$, Jorge L Zeredo ${ }^{1,2}$, Mari Kimoto ${ }^{1,3}$, Fajar H Nasution ${ }^{1,4}$, Takafumi Hirano ${ }^{1}$, Kazuo Toda ${ }^{1}$

${ }^{1}$ Integrative Sensory Physiology, Graduate School of Biomedical Sciences, Nagasaki

University, Nagasaki, Japan, ${ }^{2}$ University of Brasilia, Brasilia, Brazil, ${ }^{3}$ Physiological Laboratories, Japan Women's University, Tokyo, Japan and ${ }^{4}$ Faculty of Dentistry, Trisakti University, Jakarta, Indonesia

Running title: Electroacupuncture and NRL neurons

Corresponding author: Dr. Kazuo Toda, Integrative Sensory Physiology, Graduate School of Biomedical Sciences, Nagasaki University, 1-7-1 Sakamoto, Nagasaki, Nagasaki 852-8588, Japan.Email: k-toda@nagasaki-u.ac.jp 


\section{ABSTRACT}

A descending inhibitory mechanism from the periaqueductal gray (PAG) to the spinal cord through the nucleus raphe magnus (NRM) is strongly involved in endogenous analgesic system produced by acupuncture stimulation. In addition to the PAG to NRM system which descends in the medial pathway of the brain stem, the nucleus reticularis lateralis (NRL) situated in the lateral part of the brain stem is reported to play an important role in modulating centrifugal antinociceptive action. In the present study, to clarify the role of NRL in acupuncture analgesia, we investigated the response properties of NRL neurons to acupuncture stimulation. The majority of NRM-projecting NRL neurons were inhibited by electroacupuncture stimulation. This effect was antagonized by ionophoretic application of naloxone, indicating that endogenous opioids act directly onto these NRL neurons. By contrast, about half of spinal projecting NRL neurons were excited by electroacupuncture stimulation, suggesting that part of the NRL neurons may modulate pain transmission directly at the spinal level.

\section{Keywords}

Electroacupuncture, nucleus reticularis lateralis, nucleus raphe magnus, spinal cord, pain, rat. 


\section{INTRODUCTION}

A descending inhibitory mechanism from the periaqueductal gray (PAG) to the spinal cord and through the nucleus raphe magnus (NRM) is strongly involved in the endogenous analgesic system produced by acupuncture stimulation (Han and Terenius,1982, Kim, et al., 2006). Direct evidences of descending inhibition in acupuncture analgesia are the facts that acupuncture stimulation can modulate neuronal activities of the NRM neurons (Toda, 1982), and that analgesic effects induced by acupuncture stimulation are attenuated after NRM lesion (Du and Zhao, 1976, Zhao, 2008).

In addition to the above- mentioned PAG to NRM system which descends in the medial pathway of the brain stem, the nucleus reticularis lateralis (NRL), situated in the lateral part of the brain stem, is reported to play an important role in modulating centrifugal antinociceptive action (Sandkuhler, et al., 1988, Murphy and Behbehani, 1993a). Hall et al. (1982) and Morton et al. (1983) reported the functional contribution of the NRL in the nociceptive modulatory system, showing that electrical stimulation of the NRL alleviates selectively nociceptive responses from the dorsal horn of the spinal cord (SC) in the cat. Antinociceptive effects evoked by NRL stimulation on the behavioral responses were reported by Gebhart and Ossipov (1986), who showed that the tail-flick reflex was fairly suppressed by electrical or glutamate stimulation of the NRL in rats. These evidences suggest 
that excitation of NRL neurons can provoke the analgesic effects through descending pathways to the SC. Therefore, regarding descending antinociceptive mechanisms in acupuncture analgesia, it is probable that acupoint stimulation could induce modulation of analgesia-related NRL neurons.

Histological studies showed that NRL neurons have complex input-output relations from-to various parts of the central nervous system (Janss and Gebhart, 1987, Pretel, at al., 1988, Murphy and Behbehani, 1993a, 1993b). It is thought that important descending analgesic mechanisms underlying acupuncture analgesia work through connections from NRL to NRM and from NRL to SC. Therefore, in the present study, to clarify the role of NRL in acupuncture analgesia, we investigated the response properties of NRL neurons after acupuncture stimulation and compared the response patterns of NRM- projection (NRL-NRM) and SC-projection NRL (NRL-SC) neurons.

\section{Methods}

The methods described here follow the ethical guidelines and received approval by the Animal Welfare Committee of Nagasaki University.

Fourty-four female Wistar albino rats weighing about $300 \mathrm{~g}$ were used in the present study. 
The animals were lightly anesthetized with thiamylal sodium (Isozol, Nippon-Iko Pharmacy, Toyama, Japan), given initially $80 \mathrm{mg} / \mathrm{kg}$ intraperitoneally and additionally $10 \mathrm{mg} / \mathrm{kg}$ intravenously as needed throughout the experiment via a cannula inserted into the femoral vein. Respiratory conditions, blood pressure, and rectal temperature were continuously monitored and maintained within normal physiological levels throughout the recording session.

The brain surface was exposed by removing the skull over NRM and NRL and covered with liquid paraffin. The stereotaxic coordinates for NRM (AP: 9.3-11.1 mm caudal to bregma, L: 0-0.3 $\mathrm{mm}$ from the midline:1.0-2.4 mm ventral to brain stem surface), and for NRL (AP: 13.2-14.4 mm caudal to bregma, L: $0.5-1.8 \mathrm{~mm}$ from the midline, D:1.5-2.2 $\mathrm{mm}$ ventral to brain stem surface) were taken from the brain atlas of Swanson (2003). In addition, the dorsal surface of the spinal cord was exposed at C6-Th3 and covered with liquid paraffin.

Single unit recordings were made from left or right NRL neurons with a two-barrel glass microelectrode filled with $2 \mathrm{M}-\mathrm{NaCl}$ and $0.1 \mathrm{M}$ naloxone hydrochloride (Sigma, N-7758). Electrical signals were amplified by a biological amplifier (DAM-80, WPI Instruments, Sarasota, USA) and instantaneous firing frequencies were continuously recorded on a chart recorder (SEN-3, NEC-Sanei, Tokyo, Japan). 
To test whether changes in NRL activities were induced by the endogenous opioid system or not, Naloxone was injected microionophotetically for $30 \mathrm{~s}, 60 \mathrm{~s}$ after the cessation of acupuncture stimulation, by passing an anodal current of 40-60 nA (IP-600, Physio-tech, Tokyo, Japan).

Projection neurons to NRM or to SC were identified by antidromic activation from electrical shocks applied to the ventral part of the NRM or dorsal horn of the SC through a concentric needle electrode (outer diameter: $1.0 \mathrm{~mm}$, inner diameter: $0.1 \mathrm{~mm}$ ) (Fig. 1). Collision test was performed to examine whether the unit being recorded projected to the NRM or to the SC (Fig. 1A and B). In addition, high frequency $(200 \mathrm{~Hz})$ stimulation evoked spike responses, indicating that the recorded spikes were antidromic.

Cathodal electroacupuncture stimulation was delivered to bilateral Ho-Ku (Large Intestine 4) by inserting stainless steel needles $(0.2 \mathrm{~mm}$ in diameter $)$ to a depth of $2.0 \mathrm{~mm}$. An anodal silver-plate electrode (30 mm x $30 \mathrm{~mm}$ ) was placed on the center of the abdomen. Rectangular constant current pulses of $0.1 \mathrm{~ms}$ in duration were delivered at $45 \mathrm{~Hz}$ for $15 \mathrm{~min}$. The intensity of electroacupuncture stimulation was the one with which moderate muscle contraction was evoked very near to the Ho-ku point. These intensities (ranging from 40 to 70 $\mu \mathrm{A})$ were about 5 times the threshold for evoking a minimum twitch. In the present study, 
application of electroacupuncture stimulation was applied in one rat one to three times, as we have previously showed that the effects of electroacupuncture are reproducible through repeated trials (Toda, et al., 1980).

The sites of recording in the NRL were histologically identified by making an electrical lesion using a 2-mA DC current passing for $2 \mathrm{~min}$. At the end of experiment, the rat was killed by an overdose of thiamylal sodium.

The paired t-test was used to determine the statistical significance of the data before and after electroacupuncture stimulation. Mean ongoing activity $60 \mathrm{~s}$ just before and just after electroacupuncture stimulation were compared in each unit, using the same statistical analysis as reported by Hirano, et al (2008). 1-SD variation was checked to distinguish TYPE E (acupuncture-induced excitation), TYPE I (acupuncture-induced inhibition) and TYPE O (no change) in NRL neurons. Statistical significance was set at the 5\% level $(\mathrm{P}<0.05)$. The software Statview (version 5.0, SAS Institute, Cary, NC) aided in statistical analysis.

\section{RESULTS}

Data were collected from a total of 77 NRL neurons. Twenty-seven neurons had projection to NRM (NRL-NRM neurons) and 50 neurons had descending projection to SC (NRL-SC 
neurons) (Table 1). Neurons having projections to both NRM and SC were not found in the present study.

\section{Responses of NRL-NRM neurons}

The firing pattern of spontaneously active NRL-NRM neurons was quite irregular, as shown by the variability in instantaneous firing frequency. Unit activities of about $70 \%$ of the NRL-NRM neurons (19/27) were inhibited after electroacupuncture stimulation (TYPE I).

Figure 2 shows a typical example of ongoing activities in a NRL-NRM neuron before and immediately after the cessation of 15 min-acupuncture stimulation of $\mathrm{Ho}-\mathrm{Ku}$, and the effects of ionophoretic application of naloxone (50 nA, 30s). As clearly seen, spontaneous activities were well inhibited after electroacupuncture stimulation. When naloxone was injected ionophoretically $60 \mathrm{sec}$ after the cessation of acupuncture stimulation, acupuncture-induced decrease of ongoing activities was well antagonized.

Ongoing activities of three neurons (11\%) were excited after electroacupuncture stimulation

(TYPE E). Excited responses were not affected by ionophoretic application of naloxone.

Activities of five neurons (19\%) were not changed (TYPE O) after acupuncture stimulation (Table 1). Mean firing rates before and after electroacupuncture stimulation were 3.87 and 
1.44 Hz in TYPE I, and 2.63 and $4.20 \mathrm{~Hz}$ in Type E, respectively (Fig.3, upper trace).

The highest percentage of the descending NRL-NRM neurons were found in the rostro-medial part of the NRL; however, there was no significant tendency for the distribution of the three types of NRL-NRM neurons (Fig. 4).

\section{Responses of NRL-SC neurons}

In NRL-SC neurons, there were five TYPE I neurons (10). Ongoing activities in 24 neurons (48\%) were excited (TYPE E) after electroacupuncture stimulation. Mean firing rates before and after electroacupuncture stimulation were 3.10 and $1.32 \mathrm{~Hz}$ in TYPE I, and 1.99 and 4.07 Hz in Type E, respectively (Fig. 3, lower trace). Figure 5 shows a typical example of on-going activities of the TYPE E -NRL-SC neuron before and immediately after the cessation of 15 min-acupuncture stimulation of $\mathrm{Ho}-\mathrm{Ku}$, and the effects of ionophoretic injection of naloxone (60 nA, 30s). In this neuron, naloxone did not alter acupuncture-induced neuronal responses. Forty-two percent of the NRL-SC neurons (21/50) were unaffected by electroacupuncture stimulation (TYPE O) (Table 1).

Histological investigations revealed that most NRL-SC neurons were found in the caudal-lateral part of the NRL (Fig. 6). As in NRL-NRM neurons, NRL-SC neurons showed 
no significant tendency in spatial distribution of unit types.

\section{DISCUSSION}

The present study demonstrated that the majority of NRL-NRM neurons are inhibited by electroacupuncture stimulation. These effects were antagonized by ionophoretic application of naloxone, indicating that endogenous opioids act directly onto NRL-NRM neurons. In contrast, about half of NRL-SC neurons were excited by electroacupuncture stimulation and naloxone did not affect acupuncture-induced excitation of the NRL-SC neurons. Projection from NRL to NRM or from NRL to SC was checked electrophysiologically by high frequency stimulation and collision test between spontaneous and evoked spikes. The present study is the first to report on functional differences between NRL-NRM and NRL-SC neurons in electroacupuncture analgesia.

The functional role of brain stem structures in centrifugal modulation of the spinal nociceptive relay has been the main focus of research in endogenous antinociceptive systems (Gebhart, 2004, Bee and Dickenson, 2007, Ossipov, 2009). In addition to the medial medullary nucleus, such as the pathway through the NRM, electrical stimulation in sites lateral ventral medulla also significantly attenuate nociceptive responses in the spinal cord. Among nuclei in the lateral part of the ventral medulla, the NRL nucleus is reported to 
strongly activate analgesic or cardiovascular control systems (Lima, et al., 2002, Babic and Cirielo,2004, Tjen-A-Looi, et al., 2006). The contribution of the caudal medullary NRL to descending inhibition to the spinal cord has been reported both anatomically and physiologically. Histological data indicates that some NRL neurons including A1 area (according to Dahlstrom and Fuxe, 1964) project to the spinal cord and also to the NRM (Beitz, 1982a,b). Furthermore, PAG has strong projection both to the NRM and the NRL (Lovick, 1993). These findings suggest that there are at least two pathways for the descending inhibition to the spinal cord.

The present study revealed that the NRL has two different functions in the antinociceptive system elicited by electroacupuncture stimulation: one is a direct descending effect on the spinal nociceptive transmission-neurons, the other is a modulatory effect on NRM neurons.

\section{1) Role of NRL-NRM neurons}

As stated before, NRL-NRM neurons were mainly inhibited by electroacupuncture stimulation. In an immunohistochemical analysis, part of the NRL (A1) send neurotensin-containing or noradrenaline-containing fibers to the NRM (Atweh and Kuhar, 1977). According to a study by Hammond et al. (1980), about $85 \%$ of the A1 cells provide a noradrenergic (NA) output to the NRM. This is consistent with the demonstration that 
microinjection of noradrenergic antagonists into the NRM produces hypoalgesia, indicating that noradrenalin induces inhibition of NRM neurons (Murphy and Behbehani, 1993). The inhibition of NRL-NRM neurons after electroacupuncture stimulation shown in the present study may produce disinhibition of NRM neurons by noradrenaergic transmission. This disinhibition can enhance descending inhibition through NRM to the spinal cord in electroacupuncture analgesia.

The NRL cells have been reported to contain opioid binding sites (Atweh and Kuhar, 1977, Pearson, et al. 1980). In our study, acupuncture-induced inhibition was antagonized by ionophoretic application of naloxone, suggesting that acupuncture-released endorphins may have produced this inhibition.

\section{2) Role of NRL-SC neurons}

About half of NRL-SC neurons were excited by electroacupuncture stimulation. In a previous study by Gebhart and Ossipov (1986), it is apparent that electrical stimulation of NRL can inhibit the tail-flick reflex in rats. This inhibition is produced by descending mechanisms and mediated at least in part by spinal adrenoceptors (Pertovaara, 2006). Therefore, noradrenergic fibers whose cell bodies are situated in the NRL directly inhibit pain transmitting neurons in the spinal cord. This may be due to enhanced neuronal activity of NRL-SC neurons elicited 
by electroacupuncture stimulation. Naloxone did not antagonize the

electroacupuncture-induced increase in the activation of NRL-SC neurons, indicating that opioids were not involved in this enhancement.

In conclusion, NRL has two different functional roles in acupuncture analgesia. One is the disinhibition of NRM neurons; the other is the direct descending inhibition to the spinal cord. The former can modulate descending inhibitory pathways from PAG through NRM. 


\section{REFERENCES}

1. Atweh S.F.and M. J. Kuhar. Autoradiographic localization of opiate receptors in rat brain.

II. The brain stem. Brain Res. 129:1-12, 1977.

2. Babic T. and J. Ciriello. Medullary and spinal cord projections from cardiovascular responsive sites in the rostral ventromedial medulla. J Comp Neurol. 469:391-412, 2004.

3. Bee, L.A. and A. H. Dickenson. Rostral ventromedial medulla control of spinal sensory processing in normal and pathophysiological states. Neuroscience, 147:786-793, 2007.

4. Beitz A. J. The organization of afferent projections to the midbrain periaqueductal gray of the rat. Neuroscience, 7:133-59, 1982a.

5. Beitz A. J. The sites of origin brain stem neurotensin and serotonin projections to the rodent nucleus raphe magnus. $J$ Neurosci, 2:829-42, $1982 \mathrm{~b}$.

6. Dahlström A. and K. Fuxe. Evidence for the existence of monoamine-containing neurons in the central nervous system. I. Demonstration of monoamines in the cell bodies of brainstem neurons, Acta Physiol. Scand. 232 (Suppl. 62), 1-55, 1964 
7. Du H. J. and Y. F. Zhao. Localization of central structures involved in descending inhibitory effect of acupuncture on visceral-somatic reflex discharges, Sci. Sin. 19. $137-148,1976$

8. Gebhart G. F. Descending modulation of pain. Neurosci Biobehav Rev. 27:729-37, 2004

9. Gebhart G. F and M. H. Ossipov. Characterization of inhibition of the spinal nociceptive tail-flick reflex in the rat from the medullary lateral reticular nucleus. $J$ Neurosci. 6:701-13, 1986 .

10. Hall J. G., W. A. Duggan, C. R. Morton and S. M. Johnson. The location of brainstem neurones tonically inhibiting dorsal horn neurones of the cat. Brain Res. 244:215-22, 1982

11. Hammond D. L., R. A. Levy and H. K. Proudfit. Hypoalgesia following microinjection of noradrenergic antagonists in the nucleus raphe magnus Pain. 9:85-101, 1980.

12. Han J. S. and L. Terenius. Neurochemical basis of acupuncture analgesia. Annu Rev 
Pharmacol Toxicol. 22:193-220, 1982.

13. Hirano T., J. L. Zeredo, M. Kimoto, K. Moritaka, F. H. Nasution and K. Toda.

Disinhibitory involvement of the anterior cingulate cortex in the descending antinociceptive effect induced by electroacupuncture stimulation in rats. Am J Chin Med. $36: 569-77,2008$

14. Janss A. J. and G. F. Gebhart. Spinal monoaminergic receptors mediate the antinociception produced by glutamate in the medullary lateral reticular nucleus.

J Neurosci 7:2862-73, 1987.

15. Kim J. H., Y. S. Gwak, I. Lee, I. C. Sohn, M. S. Kim, D. O. Choi, D. B. Baek and B. R. Park. Antinociceptive effects of heterotopic electroacupuncture in formalin-induced pain. Am J Chin Med. 34:565-74, 2006

16. Lima D, A. Albino-Teixeira and I. Tavares. The caudal medullary ventrolateral reticular formation in nociceptive-cardiovascular integration. An experimental study in the rat. Exp Physiol. 87:267-74, 2002 
17. Lovick T. A. The periaqueductal gray-rostral medulla connection in the defence reaction: efferent pathways and descending control mechanisms. Behav Brain Res.;58:19-25, 1993.

18. Morton CR, S. M. Johnson and A. W. Duggan. Lateral reticular regions and the descending control of dorsal horn neurones of the cat: selective inhibition by electrical stimulation. Brain Res. 275:13-21, 1983.

19. Murphy A. Z. and M. M. Behbehani. Electrophysiological characterization of the projection from the nucleus raphe magnus to the lateral reticular nucleus: possible role of an excitatory amino acid in synaptic activation. Brain Res. 606:68-78, 1993a

20. Murphy A. Z. and M. M. Behbehani. Role of norepinephrine in the interaction between the lateral reticular nucleus and the nucleus raphe magnus: an electrophysiological and behavioral study. Pain. 55:183-93, 1993b

21. Ossipov M. H., J. Lai, T. P. Malan Jr and F. Porreca. Spinal and supraspinal mechanisms of neuropathic pain. Ann N Y Acad Sci. 909:12-24, 2000 
22. Pearson J., L. Brandeis, E. Simon and J. Hiller. Radioautography of binding of tritiated diprenorphine to opiate receptors in the rat. Life Sci. 26:1047-52, 1980.

23. Pertovaara A. Noradrenergic pain modulation. Prog Neurobiol. 80:53-83, 2006

24. Pretel S., M. J. Guinan and E. Carstens. Inhibition of the responses of cat dorsal horn neurons to noxious skin heating by stimulation in medial or lateral medullary reticular formation. Exp Brain Res. 72:51-62, 1988.

25. Sandkühler J, C. Helmchen, Q. G. Fu and M. Zimmermann. Inhibition of spinal nociceptive neurons by excitation of cell bodies or fibers of passage at various brainstem sites in the cat. Neurosci Lett. 93:67-72,1988.

26. Swanson L. Brain Maps: Structure of the Rat Brain 3rd edition, Academic Press; 2003.

27. Tjen-A-Looi S. C., P. Li and J. C. Longhurst. Midbrain vlPAG inhibits rVLM cardiovascular sympathoexcitatory responses during electroacupuncture. Am J Physiol Heart Circ Physiol. 290:H2543-53, 2006. 
28. Toda K. Response of raphe magnus neurons after acupuncture stimulation in rat.

Brain Res. 242:350-3, 1982.

29. Toda K, H. Suda, M. Ichioka and A. Iriki. Local electrical stimulation: effective needling points for suppressing jaw opening reflex in rat. Pain. 9:199-207, 1980.

30. Zhao Z. Q. Neural mechanism underlying acupuncture analgesia Progress in Neurobiology 85: 355-375, 2008. 


\section{FIGURE LEGENDS}

A

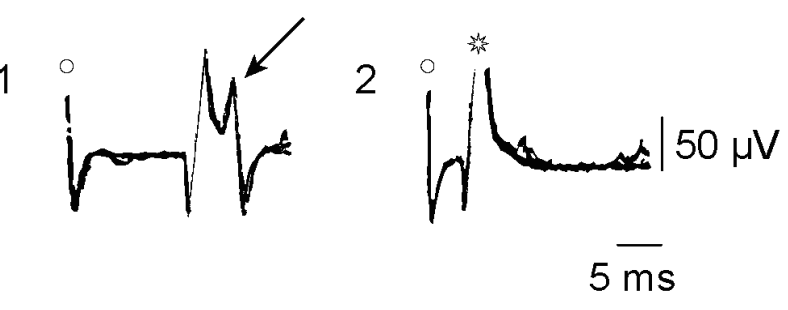

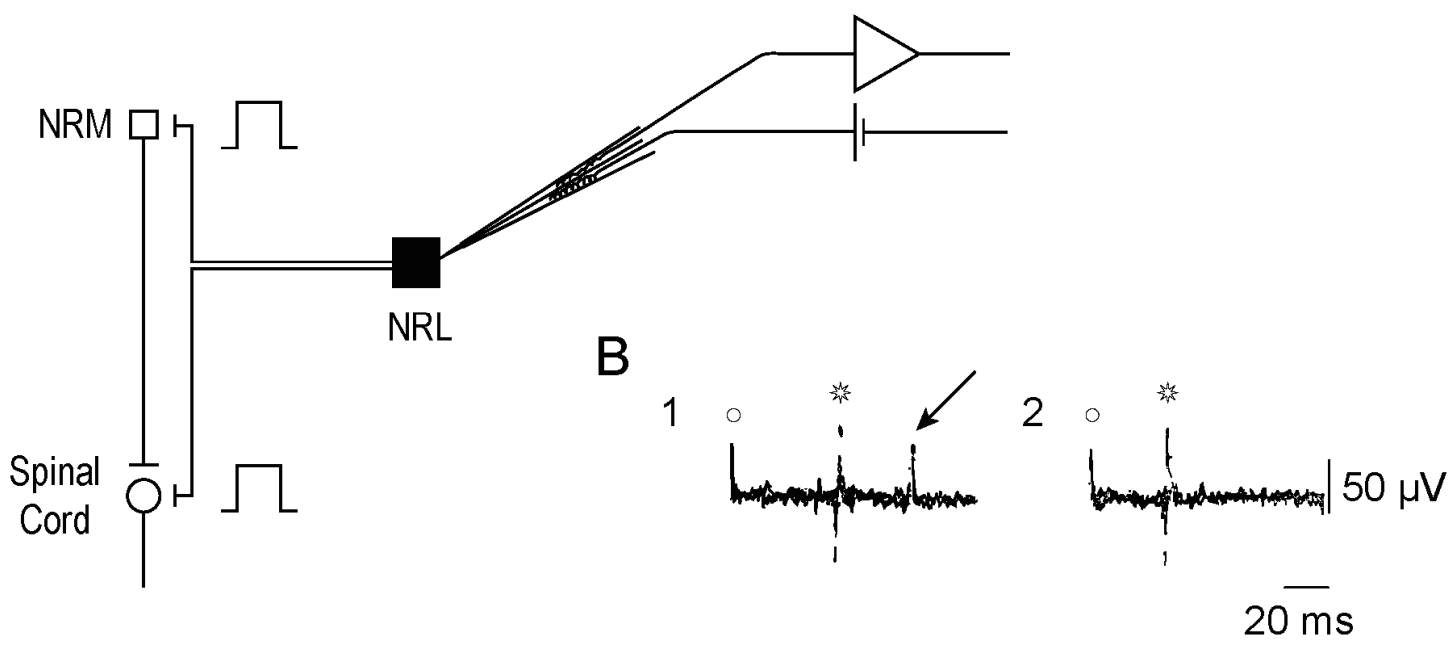

Fig.1

Schematic drawing of the experimental design. Neuronal activities of the NRL neurons were recorded by a double-barrel glass microelectrode. This electrode was also used for ionophoretic injection of naloxone. Collision test was done for NRL-NRM (A) and for NRL-SC (B) neurons, respectively. Open circles indicate spontaneous spikes; asterisks indicate stimulus artifacts; and oblique arrows indicate evoked spikes. Note that collision occurred in $\mathrm{A} 2$ and $\mathrm{B} 2$. 


\section{NRL-NRM}

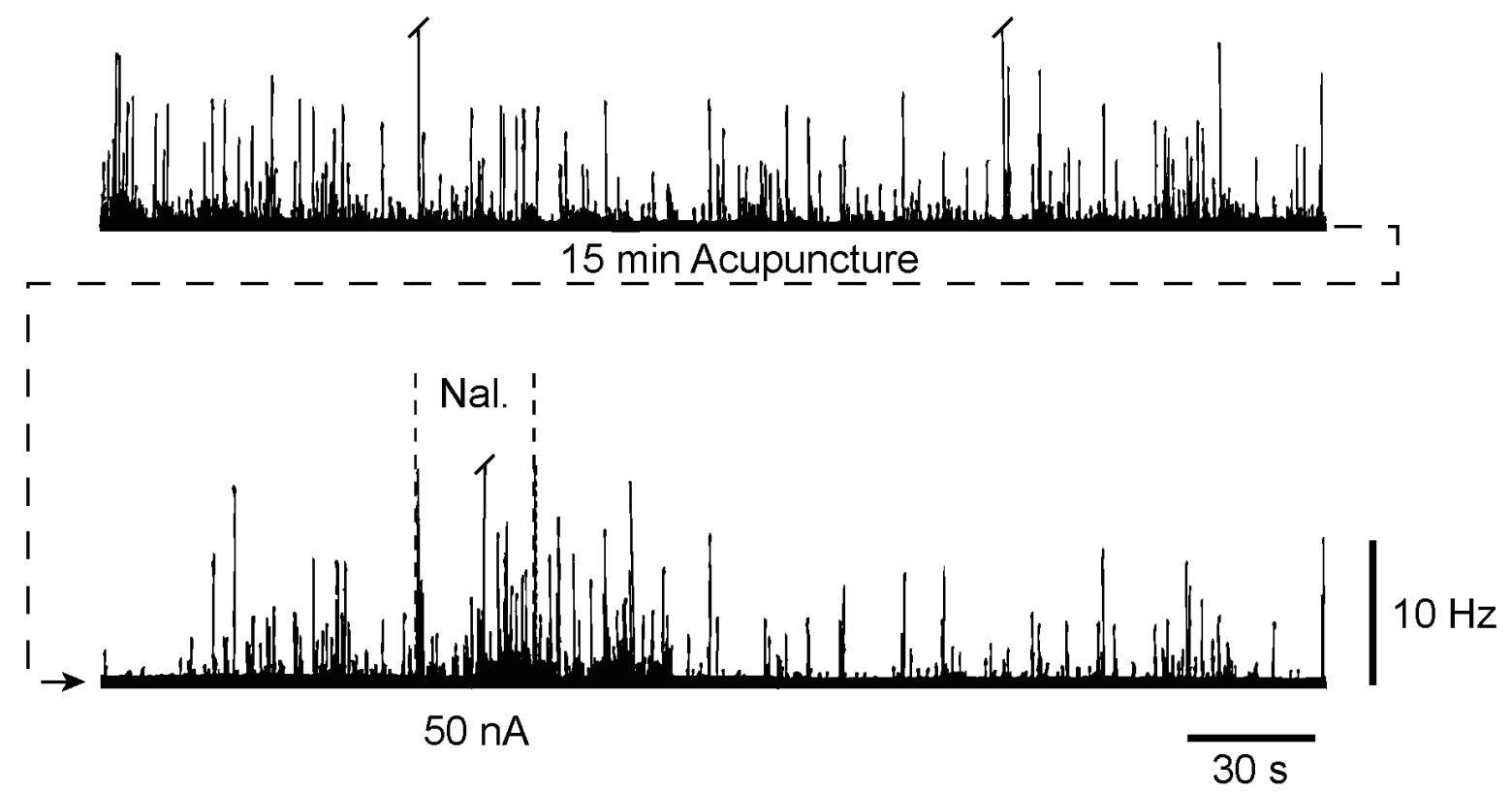

Fig. 2

Typical example of neuronal activity in a NRL-NRM neuron before (upper trace) and after

(lower trace) 15 min-electroacupuncture stimulation. The ongoing activity in this neuron was inhibited by electroacupuncture stimulation, and this inhibition was reversed by ionophoretic application of naloxone (current intensity:50 nA). Ordinate indicates instantaneous firing frequencies. 


\section{NRL-NRM}

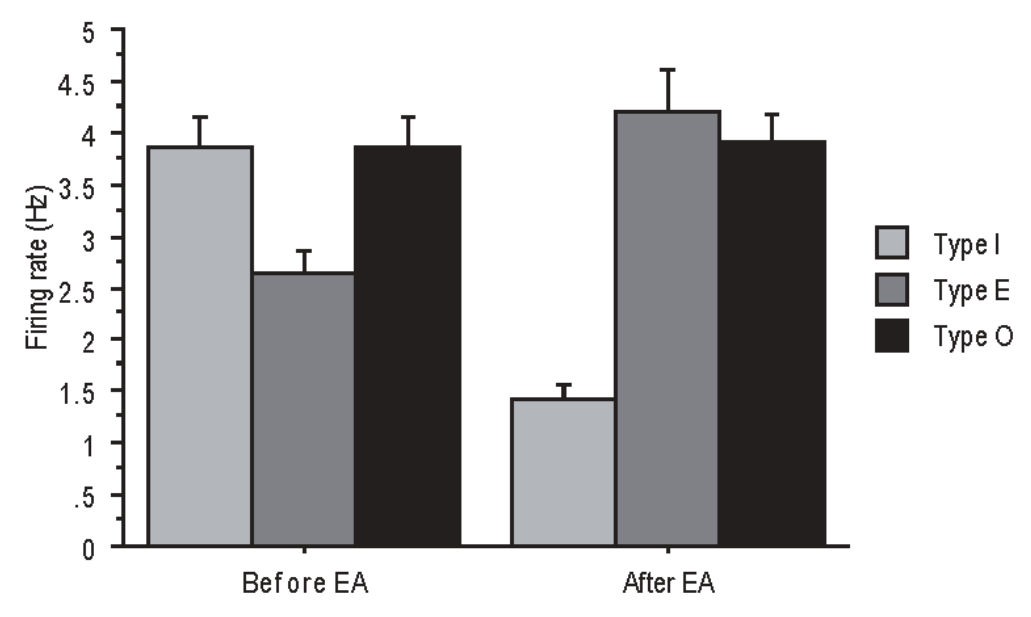

NRL-SC

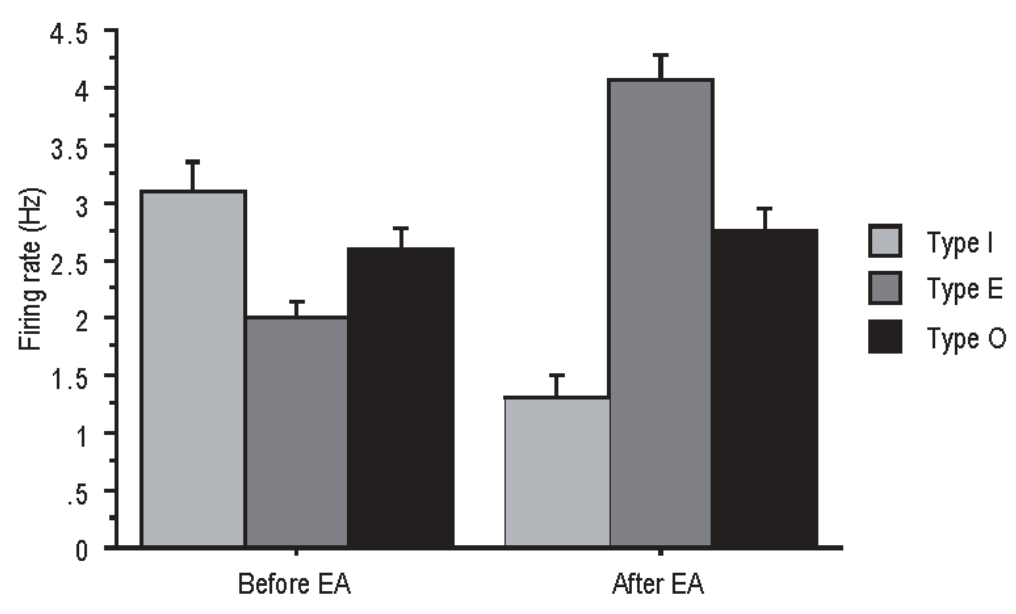

Fig. 3

Effects of electroacupuncture stimulation on the mean firing rate of NRL-NRM (upper trace) and NRL-SC (lower trace) neurons. These neurons were divided according to their responses into Type I (inhibition), Type E (excitation) and Type O (unchanged). Significant differences in firing frequencies were found between before and after electroacupuncture stimulation in Type I and Type E neurons. 


\section{NRL-NRM}

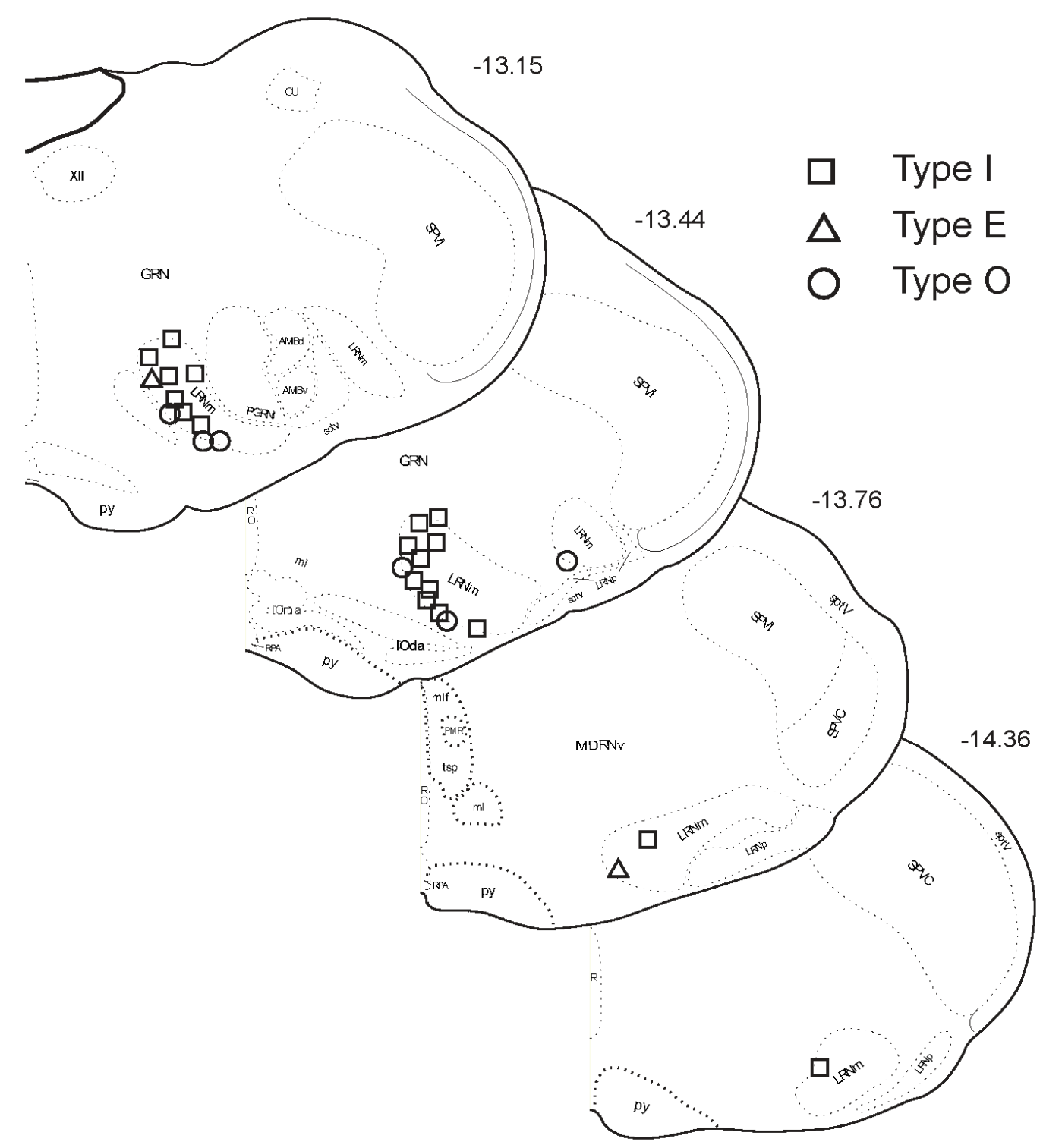

Fig.4.

Drawings of coronal brain sections showing the positions of the recording sites of the

NRL-NRM neurons. We could not distinguish any particular pattern of spatial distribution for each neuronal type. Section levels are relative to Bregma according to Swanson's brain atlas.

LRN shows lateral reticular nucleus (nucleus retucularis lateralis: NRL).LRNm shows medial part of NRL. 


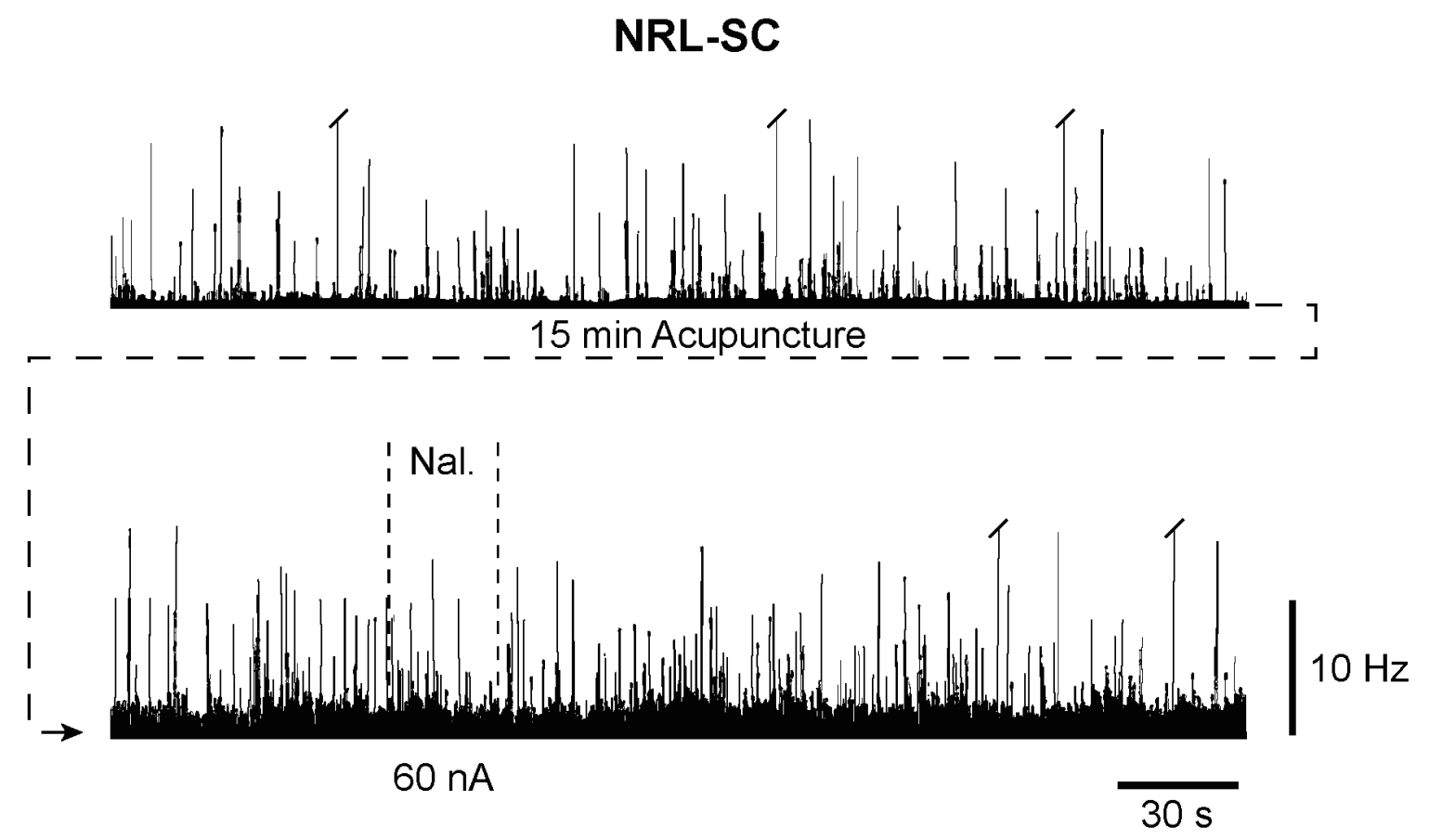

Fig.5.

Typical example of neuronal activity of a NRL-SC neuron before (upper trace) and after

(lower trace) 15 min-electroacupuncture stimulation. The ongoing activity in this neuron was increased by electroacupuncture stimulation, and this excitation was not affected by ionophoretic application of naloxone (current intensity: $60 \mathrm{nA}$ ). Ordinate indicates instantaneous firing frequencies. 


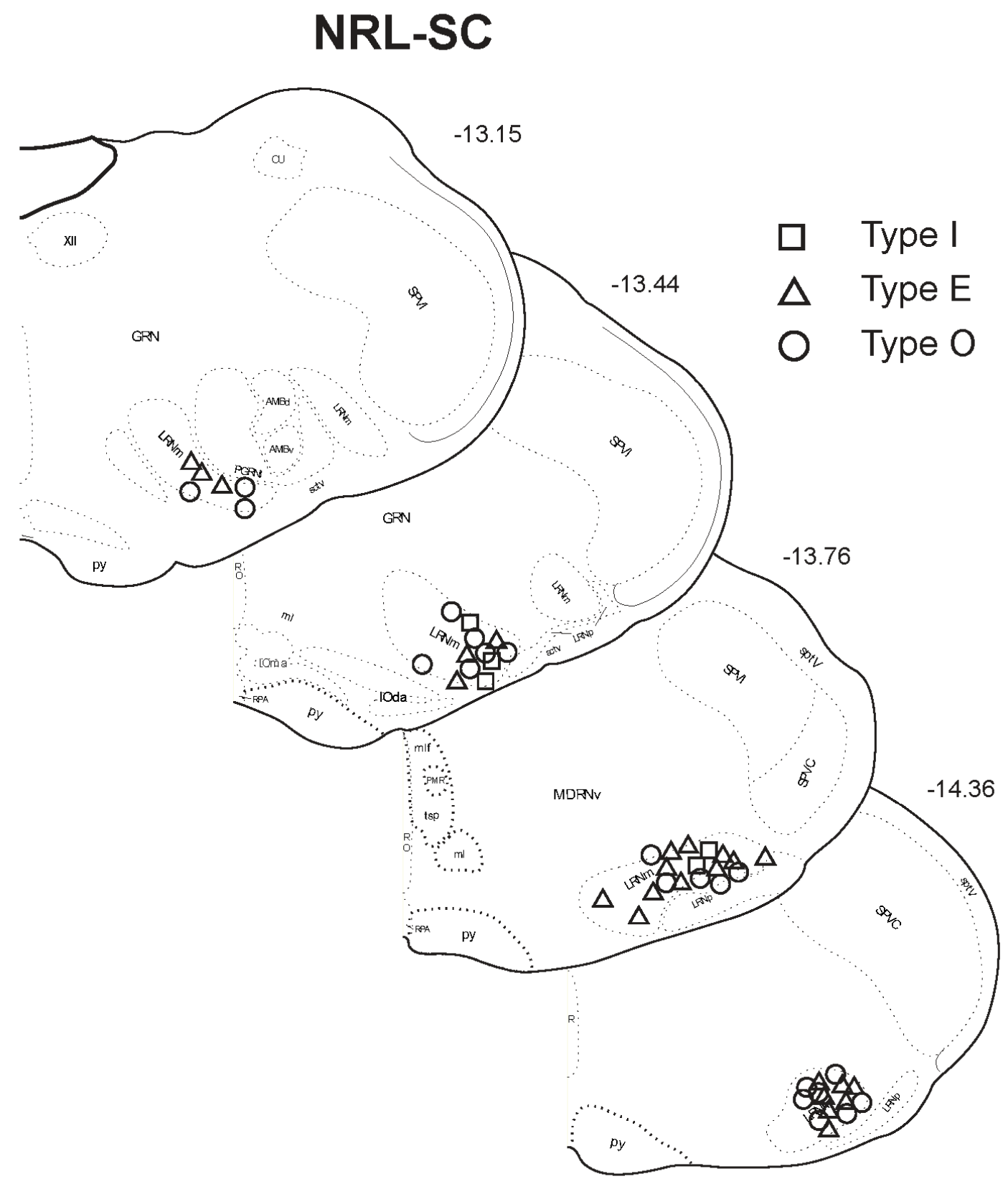

Fig.6.

Drawings of coronal brain sections showing the positions of the recording sites of the NRL-SC neurons. Most NRL-SC neurons were found in the caudal-lateral part of the NRL.

However, we could not distinguish any particular pattern of spatial distribution for each neuronal type. Section levels are relative to Bregma according to Swanson's brain atlas. 
TABLE 1. Response properties of NRL neurons to electroacupuncture stimulation (number of units by response type).

\begin{tabular}{lccc} 
& Type I & Type E & Type O \\
\hline NRL-NRM & 19 & 3 & 5 \\
NRL-SC & 5 & 24 & 21 \\
\hline Total & 24 & 27 & 26 \\
\hline
\end{tabular}

